\title{
Linx
}

Revue des linguistes de l'université Paris X Nanterre

$12 \mid 2002$

«Comme la lettre dit la vie »

\section{TRES : du multi-catégoriel au mono-catégoriel, de tresoblier trestot à sa tres chiere amie}

Christiane Marchello-Nizia

\section{OpenEdition}

Journals

Édition électronique

URL : http://journals.openedition.org/linx/1301

DOI : 10.4000/linx.1301

ISSN : 2118-9692

Éditeur

Presses universitaires de Paris Nanterre

Édition imprimée

Date de publication : 1 octobre 2002

Pagination : 178-185

ISSN : 0246-8743

\section{Référence électronique}

Christiane Marchello-Nizia, «TRES : du multi-catégoriel au mono-catégoriel, de tresoblier trestot à sa

tres chiere amie », Linx [En ligne], 12 | 2002, mis en ligne le 10 octobre 2012, consulté le 20 avril 2019.

URL : http://journals.openedition.org/linx/1301 ; DOI : 10.4000/linx.1301

Ce document a été généré automatiquement le 20 avril 2019.

Département de Sciences du langage, Université Paris Ouest 


\title{
TRES : du multi-catégoriel au mono- catégoriel, de tresoblier trestot à sa tres chiere amie
}

\author{
Christiane Marchello-Nizia
}

\section{Très : un adverbe « récent »}

1 Très est en français moderne standard le morphème privilégié destiné à intensifier adjectifs et adverbes. Mais il ne l'est devenu qu'au terme d'une série de changements qui se sont étalés sur plusieurs siècles, et dont le plus notable est certainement sa mutation catégorielle.

2 Le but de cette étude est double. Nous décrirons d'abord de façon fine les étapes par lesquelles très est passé, du latin trans(-) au moyen français tres, accomplissant plusieurs mutations entre le $11^{\mathrm{e}}$ et le $15^{\mathrm{e}}$ siècle. C'est là le complément de deux études comparables que l'on a menées sur les deux autres morphèmes d'intensité les plus courants en français standard oral et écrit, moult (jusqu'au $15^{\mathrm{e}} \mathrm{s}$.) et beaucoup (depuis le $14^{\mathrm{e}} \mathrm{s}$.), dont l'évolution est liée à celle de très (Marchello-Nizia 2000, 2000 et 2001).

3 En très ancien français, les emplois de très étaient ceux d'une préposition ou d'un préfixe. Ce sont les changements de construction, et surtout de catégorie, qu'a connus ce morphème entre la période la plus ancienne $\left(9^{\mathrm{e}}-11^{\mathrm{e}} \mathrm{s}\right.$.) et le moyen français $\left(14^{\mathrm{e}}-15^{\mathrm{e}} \mathrm{s}\right.$.), où il a formé couple avec beaucoup pour exprimer l'intensité, que nous étudions ici.

4 Le second but de cette étude est d'apporter une contribution à la réflexion actuelle sur une typologie des changements linguistiques. On se demandera si le mode de développement qu'a connu très est typique ou non : y a-t-il d'autres cas comparables?

5 Le premier aspect a fait l'objet d'un article excellent et très riche du linguiste suédois Paul Falk en 1934. Mais son étude ne porte que sur la période la plus ancienne du français : elle s'arrête en effet vers 1150, moment où est apparue la construction moderne en très + Adjectif. Pour notre part, nous irons jusqu'au moment où très aura remplacé moult dans 
une série de ses emplois. Par ailleurs, notre optique est un peu différente de celle de Falk, dans la mesure où nous porterons une attention particulière aux changements de catégorie de ce morphème, et où nous nous interrogerons aussi sur l'explication à donner à ces changements.

Le second aspect est plus complexe. Les études actuelles sur le changement linguistique, et tout particulièrement les réflexions parfois critiques sur le concept de grammaticalisation laissent percevoir qu'il existe non pas un seul type de changement linguistique (la grammaticalisation par exemple...), pas même deux types comme le pensait Meillet pour qui analogie et grammaticalisation expliquaient tout changement linguistique ${ }^{1}$, mais bien davantage sans doute. C'est dans cette réflexion pour élaborer une nouvelle typologie des changements linguistiques, et grâce peut-être en particulier à l'élaboration de la notion de 'grammaire émergente', que se situe notre démarche.

7 En ce qui concerne très, on verra qu'il s'agit d'un type de changement somme toute assez fréquent : un morphème support de plusieurs catégories grammaticales sélectionne peu à peu l'une seule d'entre elles dans son usage; on passe d'une sorte de 'syncrétisme catégoriel' (élément plurifonctionnel : particule, préfixe ou préposition) à une catégorie unique, celle d'adverbe.

\section{Très en français : préfixe, préposition, particule, ou adverbe ? Un morphème 'papillonnant ${ }^{2}$}

\subsection{Changement de catégorie}

Le morphème très est généralement analysé en français moderne comme un adverbe (ainsi : «L'intensité élevée (de l'adjectif) se marque d'abord par l'adverbe très »: Riegel, Pellat \& Rioul, p. 362). C'est sous cette catégorie que le rangent toutes les grammaires du français moderne, et également les dictionnaires.

9 Mais cette situation relativement simple et compacte est assez récente : elle date du $16^{e}$ siècle. En AF la situation est bien plus complexe et les emplois de très ${ }^{3}$ beaucoup plus variés. Le dictionnaire de Tobler et Lommatzsch (Altfranzösisches Wörterbuch, t.IX ) attribue plusieurs catégories à très: préposition mais pouvant également se combiner à d'autres morphèmes (tres dont / la / puis / ce / ci... que; tres $a$, tres devant...), ou adverbe pouvant porter sur une préposition (tres devant / desus / delez...) ou sur un adjectif ou un adverbe par ailleurs souvent 'renforcé' par une seconde particule (si tres fort, moult tres grant, plus tres haut; tres plus grant). Ce dictionnaire range par ailleurs sous des entrées spéciales les verbes préfixés en tres- (tresaler,..).

Pour Buridant (2000: p. 220-221, 497 ss., 608-609) tres est soit un préfixe, soit une préposition ( $\$ 400)$, mais son analyse est sémantique :

«En tant que préposition, tres indique un parcours vers une limite et peut s'unir à que pour former la préposition tresque, pour marquer le point d'arrivée, spatial ou temporel : tresqu'en la mer 'jusqu'à la mer' (Rol.3) ; tresqu'a ce jour 'jusqu'à ce jour' (Rol.2372). Il peut renforcer une autre préposition comme dans tres parmi, offrant la juxtaposition asyndétique de deux mots synonymes, ou encore tres en milieu, tres devant...Par association fréquente avec un terme introduisant le point final, elle en arrive à marquer le mouvement à partir d'un repère, que ce soit dans l'espace ou dans le temps, équivalant alors à dès: Le cors li trenchet tres l'un costet qu'a l'altre (Rol.1667 : 'Il lui tranche le corps de part en part') ; Tres la complie desci a l'esclairier (Moniage Guillaume II, 5568 : 'depuis complies jusqu'au point du jour'). Cf aussi en 
emploi adverbial : Et tres lors il espandy les mers et leur ordonna qu'ilz environnassent la terre Dieu (Oisivetés JA, III, 12 : 'Et dès ce moment il fit se répandre les mers..') ; Tres or en avant 'désormais' ; tres puis celle chose que 'depuis cet événement' (TL IX 59). »

11 Pour le même auteur, tres est par ailleurs catégorisé comme adverbe d'intensité pour exprimer la superlation ( $(178$, p. 220$)$, et mis sur le même plan que assez, fort, moult, trop, bien, mais sans qu'un lien soit établi avec les autres emplois. Soulignons par ailleurs une différence non négligeable: en tant que préposition, ce morphème apparaît sous deux formes: souvent tries / triers, parfois tres, alors que comme adverbe ou préfixe on ne rencontre que la seconde forme.

\subsection{La nécessité d'une analyse diachronique}

12 La description donnée par Buridant correspond à la conception toute synchronique et unifiée de sa Grammaire. Nous pensons que, concernant très, cette période est analysable diachroniquement: d'une part il y a, pensons-nous, non pas coexistence de plusieurs valeurs de très, mais au moins partiellement succession. Et d'autre part la période cruciale pour son évolution est justement celle des $12^{\mathrm{e}}-13^{\mathrm{e}}$ siècles. P. Falk avait vu un certain nombre de points, et c'est à sa suite que nous reprenons la description diachronique des constructions en très.

13 L'existence de vastes corpus et l'utilisation des outils de traitement automatiques qui les accompagnent nous a permis d'affiner cette description, et en particulier en mettant en évidence une chronologie de bien des emplois de très qui sont donnés comme synchroniques.

\section{Chronologie des emplois de tres}

\subsection{Tres au $10^{\mathrm{e}} \mathrm{s}$. : trestot}

Dans les plus anciens textes, Serments de Strasbourg, Séquence de sainte Eulalie, Sermon sur Jonas, par ailleurs fort brefs, il n'y a aucune occurrence de tres. Les premiers emplois se trouvent dans deux textes un peu plus tardifs, composés vers l'an mil à la jonction des langues d'oïl et d'oc, la Passion de Clermont et la Vie de saint Léger. Il s'agit de trestot, forme intensifiée de tot : 'absolument tout / tous'.

\subsection{Tres vers 1050 : tresbien tresoblïer trestot...}

15 Un demi-siècle plus tard, dans la Vie de saint Alexis du manuscrit de Hildesheim, une série d'emplois nouveaux de tres apparaissent : ce sont, outre trestot qui reste employé jusqu'au $15^{\mathrm{e}} \mathrm{s}$. mais avec une fréquence décroissante depuis le $14^{\mathrm{e}} \mathrm{s}$., les emplois de préposition ( tres l'us, tres sei: 'près de', 'tout près de', ou 'derrière soi'), de préfixe de l'adverbe bien ( tresbien), ainsi que celui de préfixe verbal 'perfectif' marquant qu'une action est totalement achevée :

La dreite vide nus funt tresoblier (Alexis 619: 'Ils nous font totalement oublier la véritable vie')

16 «Ço'st cil qui tres l'us set. » (Alexis 178: 'C'est celui qui se trouve tout près? derrière? juste au-delà ? du seuil') 
17 Tres sei la tint, ne la volt demustrer (Alexis 286 : 'Il la tint tout contre lui ? par devers lui? il ne voulait pas la montrer')

18 Le préfixe verbal se montre très vite très productif: une liste non exhaustive des verbes ainsi préfixés et attestés aux $12^{\mathrm{e}}$ et $13^{\mathrm{e}} \mathrm{s}$. l'illustre, dont bon nombre se trouvaient d'ailleurs déjà sous leur forme latine dans les Gloses : d'abord dans les plus anciens textes tresoblier, trespasser, tressaillir, trescurre (transcurrere), tresturner, tressuer, puis dès le milieu $\mathrm{du} 12^{\mathrm{e}} \mathrm{s}$. tresaler (trans alaret), trespenser, tresprendre, tresjeter, trespercier, tresnoer, tresbuschier, tresveoir, treschangier, tresporter, trescouper, tresfichier, tresfiner, tresformer (transformare), tresför, tresluire, tresmener, tresmordre, tresmuer (transmutare), tresoï, trespartir, tresposer, trestuer, tresvaser, tresvirer, tresvoler (cf TL IX 598-642). Mais après le $13^{\mathrm{e}}$ $\mathrm{s}$., on ne rencontre guère de nouveaux verbes ainsi formés, signe que la productivité du suffixe devient nulle.

\subsection{Début du $12^{\mathrm{e}} \mathrm{s}$. : les prépositions-particules tres et tresque : une origine anglo-normande?}

19 Au début du $12^{\mathrm{e}} \mathrm{s}$., tres- voit de nouveaux emplois se développer dans plusieurs textes de l'aire anglo-normande. La Chanson de Roland, texte anglo-normand composé vers l'an 1100, et Gormont et Isembart, chanson de geste de la même période, composée entre 1100 et 1130 dans le centre de la France mais copiée par un scribe anglo-normand, ajoutent aux emplois de tres préfixe verbal et préposition la fonction de préfixe de préposition ayant valeur de description d'un parcours entre la source d'un mouvement et sa cible (nous reprenons la notion de 'cible' à Claude Vandeloise (1986) mais préférons celle de 'source' à 'site' qu'il emploie, moins propice au déplacement); tres porte aussi une valeur plus abstraite, celle d'un parcours sémantique, marquant la perfection d'une qualité ou d'un trait : 'juste' ; selon l'option choisie, on aboutit à deux traductions différentes :

La teste en fist voler a destre, Tres devant li, sur la bel herbe (Gormont 56 :'Il fit voler sa tête (celle de son ennemi) à droite juste/jusque ? devant lui, sur la belle herbe')

Mort le tresturnent tres enmi un guaret. (Roland 1385: 'Ils l'abattent mort et le précipitent ( ?) dans un guéret')

20 Tant l'emploi prépositionnel de tres / tries que son emploi pré-prépositionnel se révèleront éphémères, puisqu'on ne les rencontre plus après le $13^{\mathrm{e}} \mathrm{s}$. L'un comme l'autre, au $12^{\mathrm{e}}$, entrent dans un nombre de constructions limité. Tres préposition locale ne s'emploie que dans un petit nombre de contextes: tres sei/lui/aus 'derrière'(guarder / laissier / mener), tries le dos (les mains / les poinz liés...), ainsi que dans l'expression tres un tres autre ('l'un derrière l'autre'). Dans ses emplois temporels, un peu plus tardifs (mi-12 s.), tres se limite également aussi à un petit nombre de contextes :

Qui m'at apris a chanter tres m'anfance (Conon de Béthune, Chansons 52 :..'qui m'a appris à chanter durant ? dès ? depuis ? mon adolescence')

21 A la même époque et dans la même région, Philippe de Thaon dans son Comput (composé en Angleterre en 1113 ou en 1119) introduit deux nouvelle formes dérivées : tresque, enemploi prépositionnel, et detrés / detriés en emploi adverbial (mais dès le milieu du $12^{\mathrm{e}}$ s. detrés sera préposition, et déjà chez Philippe de Thaon, dans le Bestiaire, cet adverbe s'était lexicalisé pour désigner l'arrière-train des animaux) :

...Sagittarie, Ço est beste...de humaine figure tresque la ceinture,

E detrés ad faiture De cheval... (Comput 1404-6: 'Le Sagittaire, c'est une bête ayant

forme humaine jusqu'à la ceinture, et derrière il a forme de cheval')

Kar humaine figure Ad tresque a la ceinture (Comput 1729-30) 
Un emploi de tres corrélé à que dans la Chanson de Roland se révélait déjà fort proche de ce tresque:

Le cors li trenchet tres l'un costet qu'a l'altre, (Roland 1506 : 'Il lui tranche le corps d'un côté jusqu'à l'autre / de part en part')

Dans le même Roland un troisième emploi de tresque apparait: celui de subordonnant uniquement temporel marquant le moment cible de perfectivité du procès principal dans le futur comme dans le passé : 'jusqu'à ce que' :

Fait cels guarder tresque li dreiz en serat. (Roland 3854 : 'Il les fait garder jusqu'à ce que le jugement ait lieu')

La noit demurent tresque vint al jur cler. (Roland 162 : 'Ils restent là la nuit, jusqu'au lever du jour clair')

Toujours dans cette période du début du $12^{\mathrm{e}} \mathrm{s}$., et toujours en anglo-normand, Benedeit, l'auteur du Voyage de saint Brendan (composé en 1121 au plus tard) offre le plus ancien emploi de tres- comme préfixe nominal dans le substantif tresturn:

En la roe sui en tresturn (Brendan 1354 : 'Je tourne attaché à la roue')

\subsection{Tres chiere, tres douce dame... : tres + adjectif ou adverbe : subjectification? emphase?}

25 Au milieu du $12^{\mathrm{e}} \mathrm{s}$., mais cette fois-ci d'abord dans des textes composés dans la région d'Ile-de-France semble-t-il, apparait un nouveau type d'emploi pour tres: celui de préfixe marqueur d'intensité (de superlation) pour les adjectifs et les adverbes.

Jusque là, seul bien pouvait être ainsi préfixé. A partir de 1130-1150, dans des textes de genres littéraires différents, tant en vers qu'en prose, dans le Couronnement de Louis (écrit vers 1130-1150 en Ile-de-France), les premières traductions anglo-normandes des Psaumes (Psautier d'Oxford ou de Montebourg: première moitié du $12^{\mathrm{e}} \mathrm{s}$.), puis le Roman de Troie de Benoît de Sainte-Maure, les Quatre Livres des Rois, et le Psautier d'Eadwin ou de Cambridge (1160), tres commence une carrière d'intensifieur de ces deux catégories de mots. Jusqu'alors, aucun texte n'offrait de tels emplois. Dans le Couronnement de Louis, les deux adjectifs concernés sont bon et fier, qui sont également dans ce texte intensifiables par molt, et l'adverbe est fort:

Nuls ne s'i claime que tres bon dreit n'i ait. (Couronnement de Louis 32 : 'Personne ne porte une accusation sans être sûr d'être parfaitement dans son bon droit')

Vers orgoillos se faiseit molt tres fiers (Couronnement de Louis 1931: 'Envers les orgueilleux il se comportait avec beaucoup d'agressivité')

Envers Guillelme l'a lancié si tres fort. (Couronnement de Louis 950 : 'Il l'a lancé avec une force si extraordinaire vers Guillaume..')

Que l'emploi emphatique est sans doute à l'origine de cette utilisation de tres apparaît dans le fait que beaucoup des occurrences de tres + adjectif dans les premiers textes où ce tour apparaît sont en fait des doubles intensifications, comme ci-dessus dans le Couronnement et chez Chrétien de Troyes; dans le Couronnement deux occurrences sur quatre sont de ce type, une sur six dans les Quatre Livres des Rois, et presque toutes les occurrences de tres devant adjectif dans le Roman de Troie de Benoît de Sainte-Maure également. Ces adjectifs sont souvent les mêmes : tres douce dame, tres chier, etc. Falk avait montré que dans les traductions-adaptations du latin, c'est tres bien plus souvent que molt qui était choisi pour rendre le superlatif en -issimus; or ce superlatif est un évaluatif emphatique. 
Le groupe des adjectifs désormais intensifiables par tres et non plus seulement par moult s'accroît peu à peu, mais lentement : dur, grant sont à leur tour préfixables en tres- ; puis dans les Quatre Livres des Rois (vers 1160) on a haut, saive, petit, fin. Mais jusqu'au $15^{\mathrm{e}} \mathrm{s}$. la liste de ces adjectifs n'augmentera pas significativement: mout reste l'intensificateur privilégié des adjectifs longs et postposés, ainsi que des attributs car avec moult la tmèse est possible (Mout est granz).

L'époque relativement tardive à laquelle cet emploi d'intensificateur d'adjectif est apparu (deuxième tiers du $12^{\mathrm{e}} \mathrm{s}$. dans l'état actuel de nos documents) fait supposer que tres, marqueur de l'intensif de haut degré ou 'superlatif absolu', est, comme le pense P. Falk, « une création du français pré-littéraire ou du gallo-roman (plus) que du bas latin.» $(1934: 17)$

\section{Vers une seule catégorie?}

\subsection{Une première explication de ces changements}

Les valeurs sémantiques de ce morphème pluricatégoriel ont évolué depuis le latin trans : tres(-) continue en ancien français à indiquer la traversée de l'extérieur de la cible vers l'extérieur à travers la cible (tresporter, trespasser, trespercier), ou bien le fait de parcourir de part en part la cible de l'intérieur à partir d'un point et jusqu'à une limite, que ce soit un lieu (tresaler), une durée (tresque subordonnant) ou, de façon abstraite, une notion ( trespenser 'être complètement absorbé dans ses pensées', tressaillir, tresformer 'donner une tout autre forme'; trestuit 'tous, absolument tous'), et c'est ce sens qui lui vaut de se développer en devenant le marqueur spécifique du superlatif des adjectifs ou des adverbes.

Comme le souligne en effet à juste titre P. Falk, ce ne sont certainement pas les valeurs sémantiques de la préposition tres / tries ('derrière, tout près, de l'autre côté') qui sont à l'origine de ce nouvel emploi. Il a formulé l'hypothèse, que nous reprenons, que c'est à partir du préfixe verbal tres- qu'il faut raisonner, car dans plusieurs verbes ainsi préfixés, tres- joue le rôle de préfixe renforçant ou perfectif (tresbatre 'battre excessivement', tresboivre 'boire abondamment', tresflamber et tresluire 'briller d'un éclat extraordinaire', tresoblier 'oublier complètement', trespenser 'être très soucieux', tressentir 'ressentir fortement', trestuer 'tuer tout à fait', tresvenir 'parvenir').

Par ailleurs, ce préfixe verbal restait parfaitement identifiable comme préfixe pour les locuteurs des $8^{\mathrm{e}}-12^{\mathrm{e}} \mathrm{s}$. Falk appuie cette hypothèse sur deux faits. D'une part les Gloses de Reichenau ( $8^{\mathrm{e}} \mathrm{s}$.) offrent beaucoup d'exemples de gloses latin-latin où un verbe composé latin est rendu par un groupe 'verbe simple + particule' : entre autres, avec le suffixe trans (ex. : transfretavit: trans alaret (1132), transfretavit: trans navigavit (585), transgrediuntur: trans vadunt (585), transilivit : trans alavit (1133)). D'autres préfixes latins tels que pro-/porro (puer en a.f.), intr-/ intus (enz en a.f.), présentent une histoire comparable. La seconde raison est qu'il existe des verbes ayant également été préfixés en français en $\operatorname{tra}(n) \mathcal{S}^{-}$, mais qui eux ont gardé la forme tra- qui n'est plus sentie comme préfixe : on a donc en ancien français un contraste entre les cas où le préfixe s'est grammaticalisé au point de se lexicaliser (traverser), et les cas où le préfixe est encore une particule analysable (noer / tresnoer : 'naviguer' / 'traverser en bateau'). 


\subsection{Du préfixe à l'adverbe : un cas de réanalyse $: 12^{\mathrm{e}}-13^{\mathrm{e}} \mathrm{s}$. :}

33 A ses débuts, au $11^{\mathrm{e}}$ et au début du $12^{\mathrm{e}} \mathrm{s}$., tres- est à analyser comme préfixe, ainsi que le montrent trois faits : la graphie, la sélectivité, les possibilités de construction du groupe tres-+ adjectif ou adverbe. D'une part en effet, dans les manuscrits tres- est toujours graphié accolé au mot qui le suit et sur lequel il porte. D'autre part, sa sélectivité étroite l'empêche de se combiner librement : seuls un tout petit nombre d'adverbes et d'adjectifs peuvent être ainsi intensifiés; il s'agit d'adverbes et d'adjectifs évaluatifs (bien, fort; grant, chier, dolz, haut) et non pas descriptifs, et de l'indéfini trestot qui emphatise le sème de totalité ; ces adjectifs et adverbes sont brefs et pour la plupart monosyllabiques, enfin les adjectifs ainsi formés sont surtout épithètes et antéposés. Et surtout, le groupe formé par tres et l'adjectif ou l'adverbe peut être à son tour intensifié par les adverbes mout, plus ou si, ce qui montre qu'il forme une entité :

34 Li plus tres petiz de mes deiz plus est gros que...(QLR 3, 32 : 'Le plus minuscule de mes doigts est plus gros que ...')

et fu li plus tres biele riens c'onques veïst hom terriens (Eracle 223 : entre 1176 et 1184 : 'Et [ce nouveau-né]était la plus ravissante créature qu'on ait jamais vu sur terre')

N'a si tres fort gent come il sont. (Troie 3810 : nombreux cas avec si ou mout : 'Il n'existe personne d'aussi extraordinairement fort qu'eux.')

...mout tres grant vertu. (Chrétien de Troyes Erec 4492 : '....un très très grand courage';

et voir d'autres ex. de la même période dans T.-L., t.XII, colonnes 597 et 598)

Venir si tres droit et si tost, (Jean Renart, Escoufle 6817,écrit en 1200-1202 : '..venir si

exactement directement et si rapidement')

Mais si le groupe formé de tres et d'un adjectif peut être précédé de si, molt ou einsi, en AF du $12^{\mathrm{e}} \mathrm{s}$. l'inverse n'est pas vrai : * très molt grant ne se trouve pas, n'est pas (encore) grammatical; il ne le deviendra qu'après grammaticalisation de tres comme adverbe.

En effet, au $13^{\mathrm{e}} \mathrm{s}$., on trouve les mêmes constructions mais inversées : non plus plus tres grant, mais tres plus grant:

La tres plus grant humilité.. (Philippe de Novarre, Quatre Tens 5 : écrit vers 1265)

S'ele conoissoit le tres plus prodome qui soit...(Guillaume de Lorris, Rose 270 : écrit vers

mi-13es.)

38 Cela est le signe d'une mutation catégorielle. La nature de tres a changé : après une réanalyse réinterprétant tres dans tres-grant comme un adverbe détachable au moins partiellement, et non plus comme un préfixe soudé, ayant donc changé de catégorie, tres précède désormais le groupe plus + adjectif ou adverbe, comme ci-dessus. Mais si tres plus est désormais possible, ${ }^{*}$ tres moult ne se rencontre pas: signe que tres et moult font désormais partie du même paradigme.

\subsection{Tres et molt : de la cooccurrence à la concurrence :}

Dans la période des débuts du français, tres et moult n'étaient donc pas véritablement en concurrence. Tres pouvait par ailleurs être préposition ou préfixe verbal et nominal, et les constructions avec adjectif et adverbe dans lesquelles il entraitétaient moins nombreuses et moins souples que celles permises à moult. Ce n'était guère que pour intensifier les rares adjectifs et adverbes qui pouvaient se construire aussi bien avec l'un ou l'autre que les deux morphèmes intensifieurs paraissaient être en variation aux $12^{\mathrm{e}}-13^{\mathrm{e}} \mathrm{s}$. :

n'ert mie de mout grant aage (Queste del saint Graal p. 97) 
un viel home de tres grant aage (ibid. p. 83) quantitativement. D'une part tres devient nettement plus fréquent que moult dans la majorité des textes (Monstrelet 86 moult, 151 tres ; Saintré 49 moult, 727 très). Et d'autre part tres précède désormais des adjectifs jusque là tributaires de moult, et ils peuvent dès lors tous deux intensifier bel, dur, fort, grand, gros, haut, joyeux, noble, vaillant, ou les adverbes bien, fort, joyeusement, puissamment, radement, vaillamment. C'est le signe que la situation catégorielle de tres a basculé. Et au $16^{\mathrm{e}} \mathrm{s}$. moult cessera peu à peu d'être employé.

$$
\begin{aligned}
& \text { productif, cesse tout simplement d'être un préfixe. En conséquence, les verbes en tres- se } \\
& \text { transforment : soit tres- devient tre- et cesse d'être senti comme préfixe (trépasser, trépas, }
\end{aligned}
$$


tressaillir), soit ces verbes sont re-préfixés à neuf et prennent le préfixe calqué du latin trans- (transformer, transpercer).

La dé-catégorialisation de tres comme préposition et comme préfixe s'achève entre 1450 et 1500 , sa re-catégorialisation comme adverbe se confirme.

\section{Evolution sémantique et catégorielle : un «type »?}

\subsection{Evolution catégorielle : du multi-catégoriel au mono-catégoriel}

Le morphème tres comme on l'a vu a subi plusieurs changements et parcouru plusieurs zones catégorielles entre le $11^{\mathrm{e}}$ et le $15^{\mathrm{e}} \mathrm{s}$. Issu du morphème latin tra(n)s qui était soit préfixe verbal, nominal ou adjectival, soit préposition de sens spatial ('au-delà de', 'de l'autre côté de', 'par-dessus'), et qui avait peut-être donné une particule autonome en proto-roman suivant l'hypothèse reprise par P. Falk (car cela expliquerait que le - $a$ - latin ait pu devenir $-e-)$, tres- apparait en ancien français tout d'abord comme préfixe verbal et nominal (noms déverbaux) et comme préfixe pour tot et bien; puis seront attestés les emplois de préposition et de préfixe prépositionnel (tres parmi), et enfin de préfixe d'adjectif ou d'adverbe. Aux $12^{\mathrm{e}}$ et $13^{\mathrm{e}} \mathrm{s}$. tous ces emplois coexistent, et c'est dans le courant $\mathrm{du} 13^{\mathrm{e}} \mathrm{s}$. et dans les constructions avec adjectif et adverbe que s'accomplit un changement important, que l'on pourrait qualifier de 'dé-grammaticalisation' partielle, dans la mesure où tres- préfixe est réanalysé en un adverbe, c'est-à-dire en une catégorie morpho-syntaxique plus autonome. Et seuls les emplois ressortissant à cette catégorie subsisteront. Tres présente donc deux caractères : changement de catégorie grammaticale avec dé-grammaticalisation partielle, et réduction de plusieurs catégories à une seule.

Une telle évolution ne constitue pas un hapax grammatical. On connaît d'autres cas comparables en ancien français, de préfixes ou particules possédant d'abord plusieurs fonctionnalités et qui n'en ont conservé qu'une : ainsi par qui en ancien français était aussi bien préposition que préverbe ou particule séparable et qui n'est plus que préposition ; ainsi un très grand nombre de prépositions-adverbes qui n'ont conservé que l'un ou l'autre de ces emplois.

\subsection{Evolution sémantique : du spatial au temporel, du spatial à l'aspectuel et à l'intensif}

51 L'expansion catégorielle que connaît tres dans sa première période s'accompagne d'un changement sémantique, ainsi qu'on l'a vu. Du local au temporel comme préposition, d'où la formation de tresque essentiellement temporel, du local à l'aspectuel pour le préfixe verbal : si dans trespasser, tresporter, tresnoer, etc. la nuance apportée par le préfixe est spatiale, elle est nettement aspectuelle dans trestorner 'retourner complètement', tresoblier 'oublier totalement', treschangier 'transformer complètement', et sans doute déjà intensive dans tresluire 'étinceler' ou soi trespenser 's'absorber dans ses pensées' : du spatial marquant un parcours dynamique vers une cible ou à travers une limite, au perfectif marquant l'achèvement dynamique d'un procès, à l'intensif enfin, on peut établir une échelle sémantique parfaitement conforme aux modèles proposés en particulier dans le cadre des processus de grammaticalisation (Heine, Claudi et Hünnemeyer 1991a, développé in idem 1991b). De ce seul point de vue, l'évolution suivie par tres est comparable à la simplification sémantique qui conduit un morphème polysémique à ne 
conserver qu'une de ses valeurs, la plus abstraite ${ }^{4}$. Là encore, rien d'exceptionnel : bon nombre de morphèmes de toute nature présentent une évolution comparable.

Mais ce qui en revanche est plus intéressant, c'est de constater que tant la réduction des emplois que la réduction sémantique se situent dans la même période $\left(13^{\mathrm{e}}-15^{\mathrm{e}} \mathrm{s}\right.$.) où bon nombre d'autres morphèmes évoluent de façon comparable.

\section{BIBLIOGRAPHIE}

BUCHEGGER, H. (1890) Ueber die Präfixe in den romanischen Sprachen. Heidelberg.

BURIDANT, Claude (2000) Grammaire nouvelle de l'ancien français. Paris : SEDES.

BURIDANT, Claude (1987) « Les particules séparées en ancien français », in Cl.Buridant éd. Romanistique-germanistique : une confrontation. Presses universitaires de Strasbourg, 167-204.

BURIDANT, Claude (1995) «Les préverbes en ancien français », in A.Rousseau éd. Les Préverbes dans les langues d'Europe. Presses universitaires du Septentrion, 287-323.

FALK, Paul (1934) «Comment trans est devenu la marque du superlatif absolu en français », in Studia Neophilologica XIII : 11-44.

HEINE, Bernd, Ulrike Claudi and Friederike Hünnemeyer (1991a) « From cognition to grammar », in E.C.Traugott et B. Heine eds (1991) : 149-187.

HEINE, Bernd, Ulrike Claudi and Friederike Hünnemeyer (1991b) Grammaticalization : A conceptual Framework. Chicago : Chicago University Press.

MARCHELLO-NIZIA, Christiane (2000) «Les grammaticalisations ont-elles une cause ? Le cas de beaucoup, moult et tres en moyen français » in L'information grammaticale 87, 3-9.

MARCHELLO-NIZIA, Christiane (2000) «Le tragique destin de moult en français : changement linguistique et structures sémantico-cognitives » in Actes du XXII ${ }^{e}$ CILPR de Bruxelles.

MARCHELLO-NIZIA, Christiane (2001) «Grammaticalisation et évolution des systèmes grammaticaux ", in Langue française 130, éd. Walter De Mulder et Anne Vanderheyden : La linguistique diachronique : grammaticalisation et sémantique du prototype, 33-41.

MONSONEGO, Simone (1993) «Le développement des expressions indéfinies dans la prose narrative à la fin du Moyen Age : l'apport du Jouvencel (1460-66) », in ARBA 1 (Acta Romanica Basiliensia), 189-200.

TRAUGOTT, Elizabeth Closs and Bernd Heine (1991) Approaches to grammaticalization I et II. Amsterdam : John Benjamins Publishing Company.

WAGNER, Robert-Léon (1946) «Verbes, préfixes, adverbes complémentaires ». In Etudes romanes dédiées à Mario Roques. Paris : Droz, 207-216. 


\section{NOTES}

1. Voir A. Meillet, (1912, "L'évolution des formes grammaticales ", in Linguistique historique et linguistique générale, p. 130-148).

2. La diversité catégorielle et sémantique des formes romanes dérivées du trans / tras latin et baslatin a conduit H. Buchegger (1890 Über die Präfixe in den romanischen Sprachen, Heidelberg, p. 10) à parler de la nature 'papillonnante' ('die schmetterlingsartige Natur') de ce morphème.

3. Selon la tradition, on note ce morphème très (avec accent) pour le FM, et tres (sans accent) pour l'AF et le MF. En effet les copistes médiévaux ne connaissaient pas cet accent, et la graphie tres pour un monosyllabe implique qu'il est accentué.

4. 1991a: C'est ce que les auteurs nomment 'isolating abstraction': 'Both generalizing and isolating abstraction appear to be present as well when grammaticalization is analyzed in terms of bleaching. Lexemes become more 'abstract' by losing their semantic specificities and being increasingly reduced to their respective core meaning (generalizing abstraction) or to one particular part of their meaning (isolating abstraction).' (1991a :157)

\section{AUTEUR}

\section{CHRISTIANE MARCHELLO-NIZIA}

E.N.S. - L.S.H (Lyon)

Institut Universitaire de France

UMR 8503 « Analyses de corpus linguistiques »

GDR « Diachronie du français et évolution des langues » 